\title{
Ground state of two-component degenerate fermionic gases
}

\author{
Tomasz Karpiuk, ${ }^{1}$ Mirosław Brewczyk, ${ }^{1}$ and Kazimierz Rzążewski ${ }^{2}$ \\ 1 Uniwersytet $w$ Biatymstoku, ulica Lipowa 41, 15-424 Biatystok, Poland \\ ${ }^{2}$ Centrum Fizyki Teoretycznej PAN, Aleja Lotników 32/46, 02-668 Warsaw, Poland
}

(Dated: November 10, 2018)

\begin{abstract}
We analyze the ground state of the two-component gas of trapped ultracold fermionic atoms. We neglect the forces between atoms in the same hyperfine state (the same component). For the case when the forces between distinguishable atoms (i.e., atoms in different hyperfine states) are repulsive (positive mutual scattering length), we find the existence of critical interaction strength above which one atomic fraction expels the other from the center of the trap. When atoms from different components attract each other (negative mutual scattering length) the ground state of the system dramatically changes its structure for strong enough attraction - the Cooper pairs built of atoms in different hyperfine states appear.
\end{abstract}

PACS Number(s):05.30.Fk, 03.75.Ss

\section{INTRODUCTION}

After the first experimental achievement of quantum degeneracy in potassium ${ }^{40} \mathrm{~K}$ gas [1] followed by the cooling of other elements $\left({ }^{6} \mathrm{Li}\right)$ below the Fermi temperature [2], the interest in ultracold atomic Fermi systems has increased significantly. Since at very low temperatures the $\mathrm{s}-$ wave scattering in a single-component spin-polarized Fermi gas is excluded by the statistics, the experimental realization of quantum degeneracy requires actually trapping of two kinds of atoms. In fact, in Ref. [1] the simultaneous trapping of different spin states of ${ }^{40} \mathrm{~K}$ is reported as a way which enables cooling the fermions by rethermalizing collisions undergoing between atoms in different spin states. Another idea is to employ the sympathetic cooling technique in a mixture of fermionic and bosonic isotopes like ${ }^{6} \mathrm{Li}$ and ${ }^{7} \mathrm{Li} 2$ or even in twospecies Fermi-Bose mixtures like ${ }^{6} \mathrm{Li}-{ }^{23} \mathrm{Na}[3]$ or ${ }^{40} \mathrm{~K}-$ ${ }^{87} \mathrm{Rb}$ [4].

Several aspects of the physics of degenerate Fermi gases have been investigated in the past few years. In Ref. 5] the properties of a harmonically trapped spin-polarized (i.e., non-interacting) Fermi gas is studied by using the Thomas-Fermi approximation. This work was extended in several ways. One possibility is to consider the dipoledipole forces as in Ref. [6], where the ground state of fermionic dipoles in the normal phase is analyzed and mechanical instabilities for large enough number of particles or the dipole moment are discovered. Another way is going beyond the semiclassical approximation by including the discrete nature of the trap levels as well as by introducing the second component and the interaction between atoms from different components [7].

Not only static but also dynamic properties of cold Fermi gases have been investigated. For example, the collective excitations in degenerate fermionic gas are discussed within the hydrodynamic approximation in Ref. [8] and based on the sum rules in Ref. 9]. There has been developed an idea of generating solitons and vortices in a one-component Fermi gas in a normal phase by using the phase imprinting technique 10]. The presence of vorticity in a trapped gas rotating at low angular velocity is analyzed in Ref. [11].

Much emphasis has been also put on the achievement of superfluid phase transition. There was a proposal to reach the Cooper-paired state at temperatures comparable to the Fermi energy via a Feshbach resonance 12] for a short-range interaction between distinguishable fermions. It is plausible that the fermionic atoms confined in an optical lattice could also undergo the phase transition at high temperatures (one tenth of the free-space Fermi energy) independently of the scattering length [13]. The possibility of $\mathrm{p}$-wave pairing through the Feshbach resonance for a short-range interaction in a single-component Fermi gas was discussed in [14] whereas the achievement of the superfluid transition in dipolar gases (again in a one-component case) in Ref. [15].

Structure of binary Bose-Einstein condensates have been studied extensively over the last years [16, 17]. In Ref. 17] possible classes of solutions within ThomasFermi approximation are given and verified by numerical integration of the coupled Gross-Pitaevskii equations. For the clarity only the case when all atoms repel each other is considered. It was found that for some range of parameters (scattering lengths) the ground state of binary system is a symmetry-breaking solution. Moreover, it turns necessary to go beyond the Thomas-Fermi approximation to obtain asymmetric ground state because the contribution of the kinetic energy (neglected within Thomas-Fermi approximation) is substantial in this case.

The similar approach but for a two-component Fermi gas is reported in Ref. [18] where the role of $\mathrm{s}^{-}$and $\mathrm{p}$-wave interactions is investigated within the ThomasFermi approximation. The existence of the critical particle number was shown for pure repulsive s-wave interaction above which the spatial separation of both components appears. However, after the separation is reached, the Thomas-Fermi approach does not yield the unique answer. Further assumptions are necessary. For example, consideration of configurations with minimal interface as energetically favorable leads to asymmetric ground state of two-component Fermi gas. For attractive s-wave interaction Ref. 18] predicts the collapse of the system for 
large enough number of atoms similarly to the case of Bose-Einstein condensate.

In this paper we analyze the ground state of twocomponent trapped ultracold Fermi gas and demonstrate the existence of several characteristic coupling strengths. To this end, we use the mean-field Hartree-Fock method and also BCS related approach when necessary (in the case when different kinds of atoms attract each other). So, opposite to Ref. [18], we go beyond the ThomasFermi approximation. On the other hand, we are restricted by numerics to rather small sample of atoms. Moreover, in this paper we present results of only onedimensional calculations.

The paper is organized as follows. In Sec. II based on the Lagrange formalism, we derive the equations which govern the two-component gas of trapped ultracold fermionic atoms. Sec. III defines the mixed twoparticle density matrix and calculates densities of each component. In Secs. [V] and $[\nabla$ we discus the properties of the ground state of the system within the HartreeFock and BCS approaches. Finally, we conclude in Sec. VI

\section{EQUATIONS DESCRIBING TWO-COMPONENT FERMI GAS AT ZERO TEMPERATURE}

The time-dependent many-body Schrödinger equation can be derived as the Euler-Lagrange equation from the following Lagrangian density

$$
\begin{aligned}
& \mathcal{L}=\frac{i \hbar}{2} \Psi^{*} \frac{\partial \Psi}{\partial t}-\frac{i \hbar}{2} \Psi \frac{\partial \Psi^{*}}{\partial t}-\frac{\hbar^{2}}{2 m} \sum_{i=1}^{N} \nabla_{i} \Psi^{*} \nabla_{i} \Psi \\
& -\sum_{i=1}^{N} V_{\text {trap }}\left(\mathbf{r}_{i}\right) \Psi^{*} \Psi-\sum_{i<j} V_{\text {int }}\left(\mathbf{r}_{i}-\mathbf{r}_{j}\right) \Psi^{*} \Psi
\end{aligned}
$$

considered as a function of the many-body wave function $\Psi\left(\mathbf{r}_{1}, \ldots, \mathbf{r}_{N}\right)$ and its spatial and time derivatives. At low temperatures both components can be treated as non-interacting Fermi gases since the s-wave scattering is absent for spin-polarized fermions. The only interaction left is the repulsion or attraction between atoms of different spins. At zero temperature the wave function of two-component $(N+N$ atoms) Fermi system is assumed to be the product of two Slater determinants

$$
\begin{aligned}
& \Psi\left(\mathbf{x}_{1}, \ldots, \mathbf{x}_{N} ; \mathbf{y}_{1}, \ldots, \mathbf{y}_{N}\right)=
\end{aligned}
$$

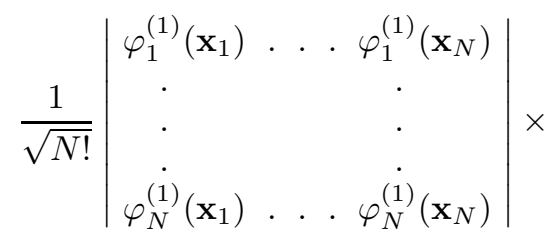

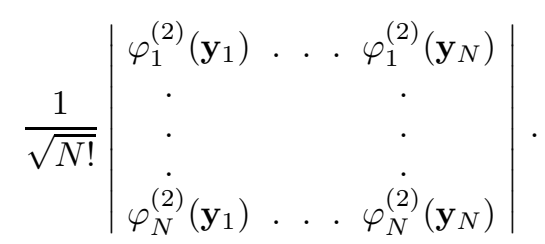

Inserting the wave function (2) into the Lagrangian obtained based on the Lagrangian density (11) and integrating each term over the appropriate $(N-1)$ coordinates for each component one obtains the effective Lagrangian density

$$
\begin{aligned}
& \mathcal{L}^{\prime}=\frac{i \hbar}{2} \sum_{i=1}^{N} \varphi_{i}^{(1) *} \frac{\partial \varphi_{i}^{(1)}}{\partial t}-\frac{i \hbar}{2} \sum_{i=1}^{N} \varphi_{i}^{(1)} \frac{\partial \varphi_{i}^{(1) *}}{\partial t} \\
& +\frac{i \hbar}{2} \sum_{i=1}^{N} \varphi_{i}^{(2) *} \frac{\partial \varphi_{i}^{(2)}}{\partial t}-\frac{i \hbar}{2} \sum_{i=1}^{N} \varphi_{i}^{(2)} \frac{\partial \varphi_{i}^{(2) *}}{\partial t} \\
& -\frac{\hbar^{2}}{2 m} \sum_{i=1}^{N} \nabla \varphi_{i}^{(1) *} \nabla \varphi_{i}^{(1)}-\frac{\hbar^{2}}{2 m} \sum_{i=1}^{N} \nabla \varphi_{i}^{(2) *} \nabla \varphi_{i}^{(2)} \\
& -V_{\text {trap }}^{(1)} \sum_{i=1}^{N}\left|\varphi_{i}^{(1)}\right|^{2}-V_{\text {trap }}^{(2)} \sum_{i=1}^{N}\left|\varphi_{i}^{(2)}\right|^{2} \\
& -g \sum_{i=1}^{N}\left|\varphi_{i}^{(1)}\right|^{2} \sum_{i=1}^{N}\left|\varphi_{i}^{(2)}\right|^{2}
\end{aligned}
$$

which depends on one--particle orbitals of both types and their spatial and time derivatives. Deriving Eq. (3) we have assumed the contact interaction between distinguishable fermions with $g$ being the coupling constant. Like in experiment [1] both atomic fractions move in harmonic potentials and the ratio of frequencies is equal to $\omega^{(1)} / \omega^{(2)}=\sqrt{9 / 7}$. Time-independent version of the Euler-Lagrange equations originating from the effective Lagrangian density (3) is given by

$$
\begin{aligned}
& -\frac{\hbar^{2}}{2 m} \nabla^{2} \varphi_{i}^{(1)}+V_{\text {trap }}^{(1)} \varphi_{i}^{(1)}+g \sum_{j=1}^{N}\left|\varphi_{j}^{(2)}\right|^{2} \varphi_{i}^{(1)}=\varepsilon_{i}^{(1)} \varphi_{i}^{(1)} \\
& -\frac{\hbar^{2}}{2 m} \nabla^{2} \varphi_{i}^{(2)}+V_{\text {trap }}^{(2)} \varphi_{i}^{(2)}+g \sum_{j=1}^{N}\left|\varphi_{j}^{(1)}\right|^{2} \varphi_{i}^{(2)}=\varepsilon_{i}^{(2)} \varphi_{i}^{(2)} \\
& i=1,2, \ldots, N
\end{aligned}
$$

with $\varepsilon_{i}^{(1)}$ and $\varepsilon_{i}^{(2)}$ being the single-particle Hartree-Fock energies. The total energy of the system is expressed in the form

$$
\begin{aligned}
& <\Psi|\mathrm{H}| \Psi>=\sum_{i=1}^{N} \varepsilon_{i}^{(1)}+\sum_{i=1}^{N} \varepsilon_{i}^{(2)} \\
& -g \sum_{i, j=1}^{N} \int\left|\varphi_{i}^{(1)}(\mathbf{x})\right|^{2}\left|\varphi_{j}^{(2)}(\mathbf{y})\right|^{2} d \mathbf{x} d \mathbf{y} .
\end{aligned}
$$

We solve numerically the set of Eqs. (4) to find the ground state of two-component degenerate fermionic gases. 


\section{ONE- AND TWO-PARTICLE DENSITY MATRICES}

Mixed two-particle density function is defined as

$$
\begin{aligned}
& \rho_{2}^{(1)(2)}\left(\mathbf{x}_{1}, \mathbf{y}_{1} ; \mathbf{x}_{1}^{\prime}, \mathbf{y}_{1}^{\prime}\right)= \\
& <\hat{\psi}^{(1) \dagger}\left(\mathbf{x}_{1}\right) \hat{\psi}^{(2) \dagger}\left(\mathbf{y}_{1}\right) \hat{\psi}^{(1)}\left(\mathbf{x}_{1}^{\prime}\right) \hat{\psi}^{(2)}\left(\mathbf{y}_{1}^{\prime}\right)>
\end{aligned}
$$

or equivalently in the position representation

$$
\begin{aligned}
& \rho_{2}^{(1)(2)}\left(\mathbf{x}_{1}, \mathbf{y}_{1} ; \mathbf{x}_{1}^{\prime}, \mathbf{y}_{1}^{\prime}\right)= \\
& \int \Psi\left(\mathbf{x}_{1}, \mathbf{x}_{2}, \ldots, \mathbf{x}_{N} ; \mathbf{y}_{1}, \mathbf{y}_{2}, \ldots, \mathbf{y}_{N}\right) \\
& \times \Psi^{*}\left(\mathbf{x}_{1}^{\prime}, \mathbf{x}_{2}, \ldots, \mathbf{x}_{N} ; \mathbf{y}_{1}^{\prime}, \mathbf{y}_{2}, \ldots, \mathbf{y}_{N}\right) \\
& \times d \mathbf{x}_{2} \ldots d \mathbf{x}_{N} d \mathbf{y}_{2} \ldots d \mathbf{y}_{N}
\end{aligned}
$$

One-particle densities are derived from mixed twoparticle density functions (6.7) by contracting over $\mathrm{x}$ or y variables

$$
\begin{aligned}
& \rho_{1}^{(1)}\left(\mathbf{x}_{1} ; \mathbf{x}_{1}^{\prime}\right)=<\hat{\psi}^{(1) \dagger}\left(\mathbf{x}_{1}\right) \hat{\psi}^{(1)}\left(\mathbf{x}_{1}^{\prime}\right)> \\
& \rho_{1}^{(2)}\left(\mathbf{y}_{1} ; \mathbf{y}_{1}^{\prime}\right)=<\hat{\psi}^{(2) \dagger}\left(\mathbf{y}_{1}\right) \hat{\psi}^{(2)}\left(\mathbf{y}_{1}^{\prime}\right)>
\end{aligned}
$$

or equivalently

$$
\begin{aligned}
& \rho_{1}^{(1)}\left(\mathbf{x}_{1} ; \mathbf{x}_{1}^{\prime}\right)=\int \rho_{2}^{(1)(2)}\left(\mathbf{x}_{1}, \mathbf{y}_{1} ; \mathbf{x}_{1}^{\prime}, \mathbf{y}_{1}\right) d \mathbf{y}_{1} \\
& \rho_{1}^{(2)}\left(\mathbf{y}_{1} ; \mathbf{y}_{1}^{\prime}\right)=\int \rho_{2}^{(1)(2)}\left(\mathbf{x}_{1}, \mathbf{y}_{1} ; \mathbf{x}_{1}, \mathbf{y}_{1}^{\prime}\right) d \mathbf{x}_{1}
\end{aligned}
$$

In the Hartree-Fock state given by (2) two-particle density matrix, since single-particle orbitals are orthogonal, reduces to

$$
\begin{aligned}
& \rho_{2}^{(1)(2)}\left(\mathbf{x}_{1}, \mathbf{y}_{1} ; \mathbf{x}_{1}^{\prime}, \mathbf{y}_{1}^{\prime}\right)= \\
& \frac{1}{N^{2}} \sum_{i, j}^{N} \varphi_{i}^{(1) *}\left(\mathbf{x}_{1}\right) \varphi_{j}^{(2) *}\left(\mathbf{y}_{1}\right) \varphi_{i}^{(1)}\left(\mathbf{x}_{1}^{\prime}\right) \varphi_{j}^{(2)}\left(\mathbf{y}_{1}^{\prime}\right)
\end{aligned}
$$

and is always a product of one-particle density matrices (two-particle correlation function vanishes)

$$
\begin{aligned}
& \rho_{1}^{(1)}\left(\mathbf{x}_{1} ; \mathbf{x}_{1}^{\prime}\right)=\frac{1}{N} \sum_{i}^{N} \varphi_{i}^{(1) *}\left(\mathbf{x}_{1}\right) \varphi_{i}^{(1)}\left(\mathbf{x}_{1}^{\prime}\right) \\
& \rho_{1}^{(2)}\left(\mathbf{y}_{1} ; \mathbf{y}_{1}^{\prime}\right)=\frac{1}{N} \sum_{i}^{N} \varphi_{i}^{(2) *}\left(\mathbf{y}_{1}\right) \varphi_{i}^{(2)}\left(\mathbf{y}_{1}^{\prime}\right)
\end{aligned}
$$

The density of each component equals the diagonal part of corresponding one-particle density matrix and for the Hartree-Fock state (2) is given by

$$
\begin{aligned}
& \rho^{(1)}\left(\mathbf{x}_{1}\right)=\frac{1}{N} \sum_{i}^{N}\left|\varphi_{i}^{(1)}\left(\mathbf{x}_{1}\right)\right|^{2} \\
& \rho^{(2)}\left(\mathbf{y}_{1}\right)=\frac{1}{N} \sum_{i}^{N}\left|\varphi_{i}^{(2)}\left(\mathbf{y}_{1}\right)\right|^{2}
\end{aligned}
$$

In Section $\square$ we show that for strong enough attraction between atoms better description of the ground state is BCS like one with significant correlations developed.

\section{POSITIVE SCATTERING LENGTH}

In this Section we consider the ground state of onedimensional two-component Fermi system with repulsive forces between atoms of different components. We solve numerically the set of Eqs. (4) (in its one-dimensional version) by using iterative procedure. At zero step we pick up the Hartree-Fock orbitals, for example as the eigenstates of the pure harmonic potentials for each component - these functions are called zero-step orbitals. Then we calculate potentials which come from the presence of other component and build Hamiltonian matrices for both parts of the system. Matrix elements are calculated in sets of basis functions which include corresponding harmonic oscillator eigenstates. The number of basis functions is typically much bigger than the number of particles $N$. At each step both matrices are diagonalized and new single-particle wave functions are obtained as well as single-particle energies. This procedure is continued until all quantities remain unchanged with prescribed accuracy.

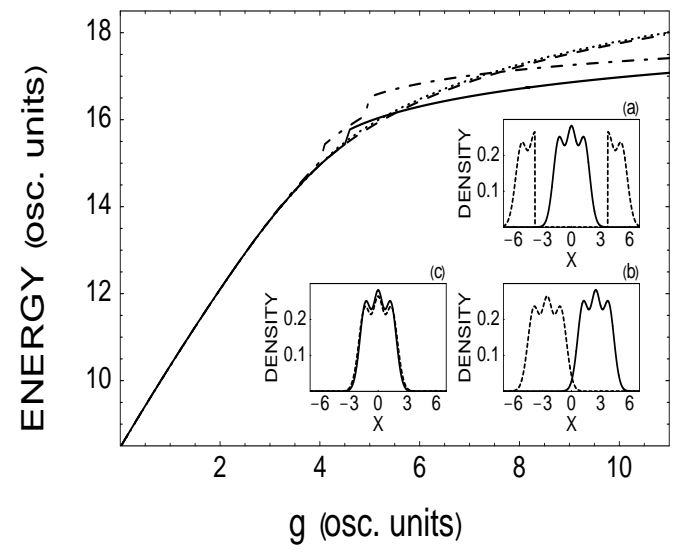

FIG. 1: Energy of $3+3$ atoms system as a function of positive interaction strength obtained within the Hartree-Fock approach. For weak enough repulsion $(\mathrm{g}<4)$ all initial conditions lead to the same density profile which is the density of the ground state. For strong repulsion the density profile of the ground state changes qualitatively its shape (see Fig. 2). Various sets of zero-step orbitals give different local minima of energy. Insets show the zero-step densities used in the numerics: (a) gives the results marked by solid line, (b) corresponds to dotted-dashed line, and (c) leads to both dotted and dashed lines. See text for further description of the procedure.

We summarize our results in Fig. [1 There exists the critical value of the interaction strength $(g \approx 4$ for $3+3$ atoms system) above which the density profile of the system changes qualitatively its shape (see Fig. 21). It is profitable from energetic point of view that the component confined in a lower frequency trap is repelled from the center of the trap. Although the potential energy is increased in such a way, the total energy, however, is de- 


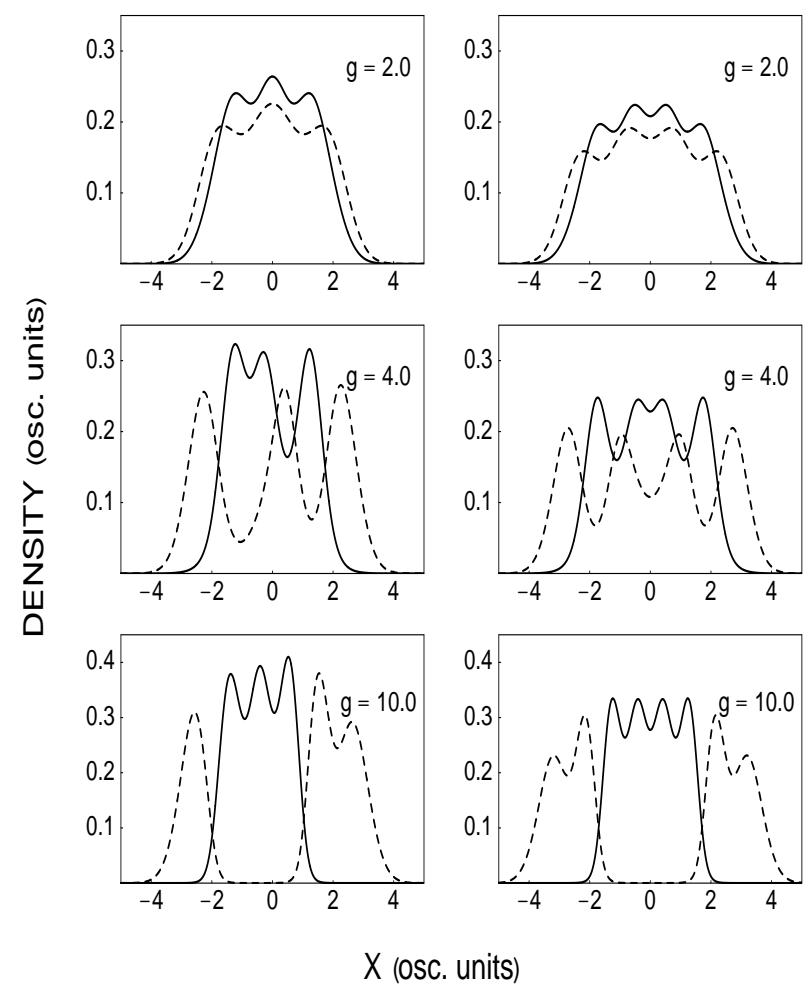

FIG. 2: Density profiles of the ground state of two-component Fermi gas for various positive interaction strengths. Left and right columns show the case of $3+3$ and $4+4$ atoms systems, respectively. For small coupling constant $(g<4)$ both fractions almost fully overlap each other whereas beyond the short intermediate interval (around $g=4$ ) fractions separate. Full curves correspond to the first (higher frequency trap) component. The broken curves indicate the second component confined in a lower frequency trap.

creased because the interaction (repulsion) is weakened. The solid line in Fig. 1 shows the energy of the ground state of two-component system. Its density is presented in Fig. 2 (left column). The fact that the density is not symmetric with respect to the center of the trap (the lowest frame) is explained by the presence of odd number of atoms in each fraction. For example, for $4+4$ atoms system the density of the ground state is symmetric (see the right column of frames in Fig. 2). The interplay between the potential and the interaction energies of the gases is even more subtle than just described. It turns out that the symmetry of the ground state strongly depends on the geometry of the traps as shown in Fig. 3 for a particular value of the coupling strength. The vertical dotted line indicates the ratio of the trap frequencies used throughout this paper. Depending on this ratio the ground state of the system switches between the asymmetric density profile (boxes in Fig. 3) like in the middle frame in Fig. 4 and symmetric one (circles in Fig. 3) as in the upper frame in Fig. 4 The vertical dashed line marks the geometry $\left(\omega^{(2)} / \omega^{(1)}=0.945\right)$ in which the ground state of the system becomes degenerate. We have checked that increasing the repulsion strength between the components the ratio $\omega^{(2)} / \omega^{(1)}$ that favors the degeneracy of the ground state is getting lower. Eventually, for $g>44$ the ground state of $3+3$ atoms system becomes asymmetric under the considered trapping potentials.

For large enough $g$ we find several local energy minima depending on the chosen set of zero-step orbitals. The lowest minimum is accessible provided one uses for the second component harmonic oscillator eigenstates which are first cut at the center and then pulled out as a zerostep orbitals (inset (a) in Fig. 1). If plain harmonic oscillator eigenstates are taken as the initial wave functions (inset (c) in Fig. 1) one follows the dashed line and eventually ends in a domain structure state (the lowest frame in Fig. (4). The dotted line matches the dashed one in Fig. 11 and was obtained by solving the Euler-Lagrange equations corresponding to the effective Lagrangian density (3) under the assumption of adiabatic approximation. Finally, the dotted-dashed line in Fig. 1 1 represents the local minimum with the densities of both fractions being shifted with respect to each other (for large enough $g$ - the middle frame in Fig. (4). This minimum is obtained when at zero step of iteration one takes shifted left (for one component) and right (for another component) harmonic oscillator eigenstates (inset (b) in Fig. 1). Nothing changes qualitatively when one goes to larger systems as shown in Fig. 5 which is the case of $50+50$ atoms system. The dense structure visible in the lowest frame of Fig. [5indicates that for much larger number of atoms we should expect a smooth overlapping distribution of both components in the corresponding case.

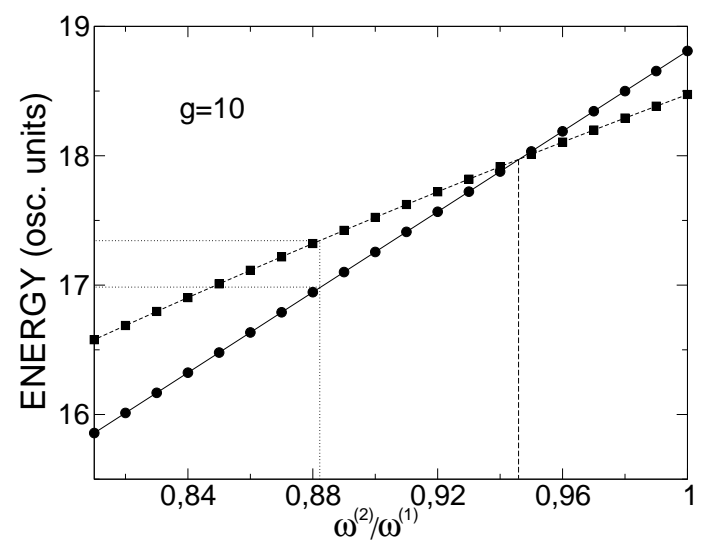

FIG. 3: The energy of an asymmetric density profile states (boxes) and symmetric ones (circles) as a function of the ratio $\omega^{(2)} / \omega^{(1)}$ for $3+3$ atoms system. The vertical dotted line shows the geometry used in this paper.

\section{NEGATIVE SCATTERING LENGTH}

In this Section we consider the ground state of twocomponent system with attractive forces between atoms of different components. First of all, we have checked 

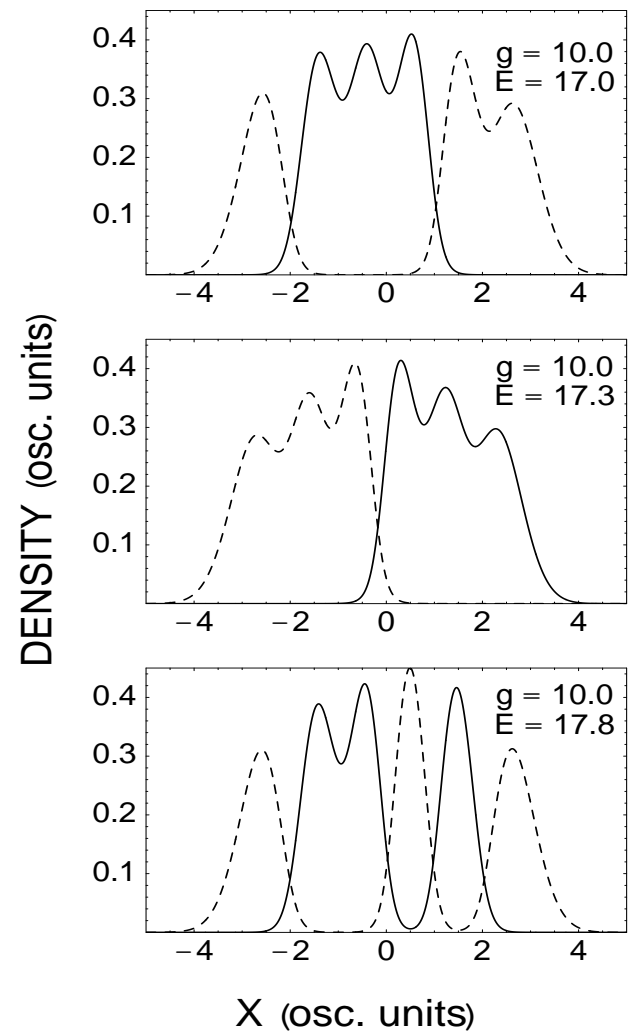

FIG. 4: Density profiles for the coupling constant $g=10$. The upper frame corresponds to the ground state whereas the middle and lower ones to the excited states which show qualitatively different patterns; separation (the middle one) and the domain structure (the lowest one).

that in this case the Hartree-Fock energies and densities of the ground state do not depend on the choice of zero-step orbitals. The resulting, ,ground state" energy is plotted as a dashed line in Fig. [6] and the densities of both fractions (for $g=-10$ ) are shown in the inset (also by dashed line). It is clear that for strong enough attraction between atoms of different fractions a new density pattern with visible fragmentation is developed. Further analysis reveals the appearance of energy gap in the single-particle Hartree-Fock spectrum (see Fig. 17). The energy gap separates off the populated Hartree-Fock levels. If one of the atoms occupying the highest level is promoted to the state just above the energy gap the total energy of the system is increased and can be calculated based on formula (51). It turns out that the surplus in energy is of the order of the energy gap. Existence of such an energy gap in a single-particle spectrum could be a signature of appearance of pairing phenomenon in a system. This possibility will be verified in the following subsection. At the same time no such gap is developed when distinguishable atoms repel each other (Fig. 8 the upper frame). Note, however, that an energy gap is also developed (Fig. 8 the lower frame) in the case of repul-
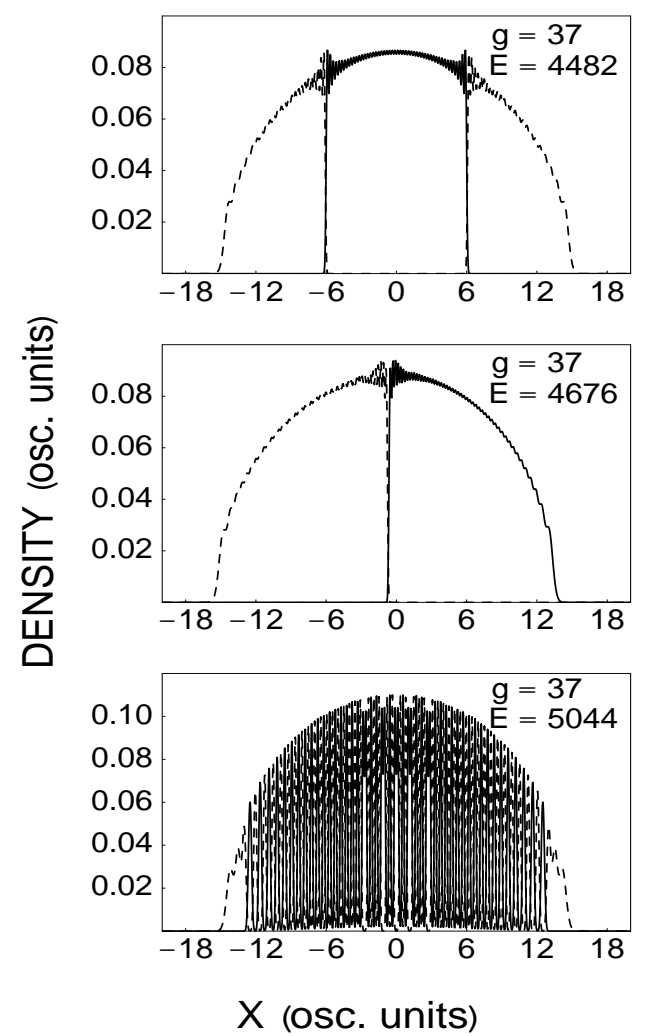

FIG. 5: The sequence of frames similar to that in Fig. 4 but for the system of $50+50$ atoms and $g=37$. Solid curves show the densities of the first component (loaded into higher frequency trap) whereas the dashed ones correspond to the second component (loaded into lower frequency trap). The lowest frame is, in fact, the case of very dense domain structure.

sion between different atoms in antiferromagnetic phase

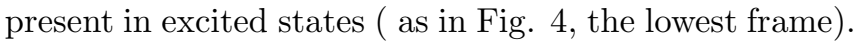

\section{A. BCS formulation}

To check the idea whether the energy gap developed in a single-particle energy spectrum is connected with the appearance of pairing phenomenon in a system, we have reformulated our problem. Within Hartree-Fock approach each particle interacts with all the others in the second component. Now, we allow for formation of pairs including atoms of different spins. More precisely, only configurations in which different atoms populate singleparticle states of the same quantum number $n$ are permitted. Hence, the ground state wave function is written in the following form 19]

$$
\left|\Psi_{\mathrm{BCS}}>=\prod_{n}\left(u_{n}+v_{n} a_{n}^{+} b_{n}^{+}\right)\right| 0>
$$




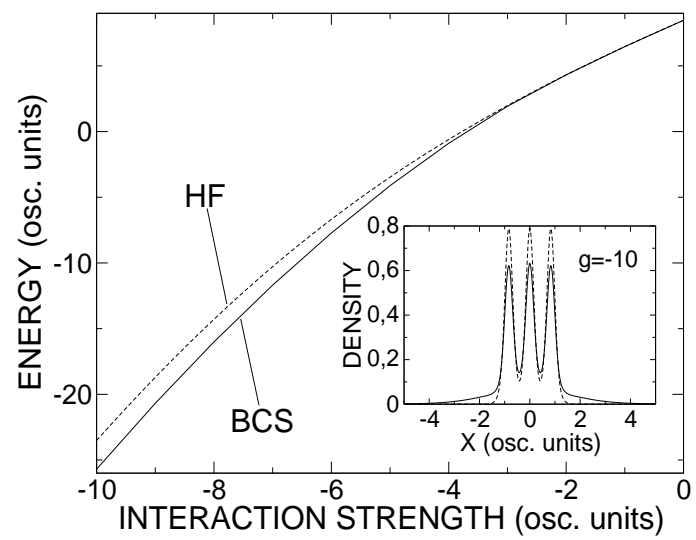

FIG. 6: Ground state energy of $3+3$ atoms system as a function of interaction strength for both the Hartree-Fock and BCS approaches. For strong enough attraction the ground state of the system shows the appearance of Cooper pairs. The inset shows the density (in oscillatory units) calculated via the Hartree-Fock (dashed line) and BCS (solid line) methods. Note that the densities for both fractions look the same.

where $u_{n}^{2}+v_{n}^{2}=1$ for each $n$ (this assures the normalization condition) and operators $a_{n}^{+}, b_{n}^{+}$create fermions, respectively, of first $\left(a_{n}^{+}\right)$and second $\left(b_{n}^{+}\right)$component. Of course, $v_{n}^{2}$ is the probability that the pair state $\varphi_{n}^{(1)} \varphi_{n}^{(2)}$ is occupied.

The Hamiltonian of the system includes kinetic and potential energies of both parts as well as the interaction between distinguishable atoms and is given by

$$
\begin{aligned}
& \mathrm{H}=\sum_{n, k}<n\left|E_{\text {kin }}^{(1)}+V_{\text {trap }}^{(1)}\right| k>a_{n}^{+} a_{k} \\
& +\sum_{m, l}<m\left|E_{\text {kin }}^{(2)}+V_{\text {trap }}^{(2)}\right| l>b_{m}^{+} b_{l} \\
& +\sum_{n, m, k, l}<n m\left|V_{\text {int }}\right| k l>a_{n}^{+} b_{m}^{+} a_{k} b_{l} .
\end{aligned}
$$

The mean value of the Hamiltonian in the ground state given by (13) is calculated as

$$
\begin{aligned}
& \mathrm{E}_{\mathrm{BCS}}=\sum_{n}\left(e_{n}^{(1)}+e_{n}^{(2)}\right) v_{n}^{2}+\sum_{n} V_{n n, n n} v_{n}^{2} \\
& +\sum_{\substack{n, m \\
n \neq m}}\left(V_{n n, m m} u_{n} u_{m} v_{n} v_{m}+V_{n m, n m} v_{n}^{2} v_{m}^{2}\right),
\end{aligned}
$$

where

$$
\begin{aligned}
& e_{n}^{(1)}=<n\left|E_{\text {kin }}^{(1)}+V_{\text {trap }}^{(1)}\right| n> \\
& e_{n}^{(2)}=<n\left|E_{\text {kin }}^{(2)}+V_{\text {trap }}^{(2)}\right| n> \\
& V_{n n, m m}=<n n\left|V_{\text {int }}\right| m m> \\
& V_{n m, n m}=<n m\left|V_{\text {int }}\right| n m>.
\end{aligned}
$$

The first term in (15) is the sum of kinetic and potential energies of all atoms, the second and third (the first
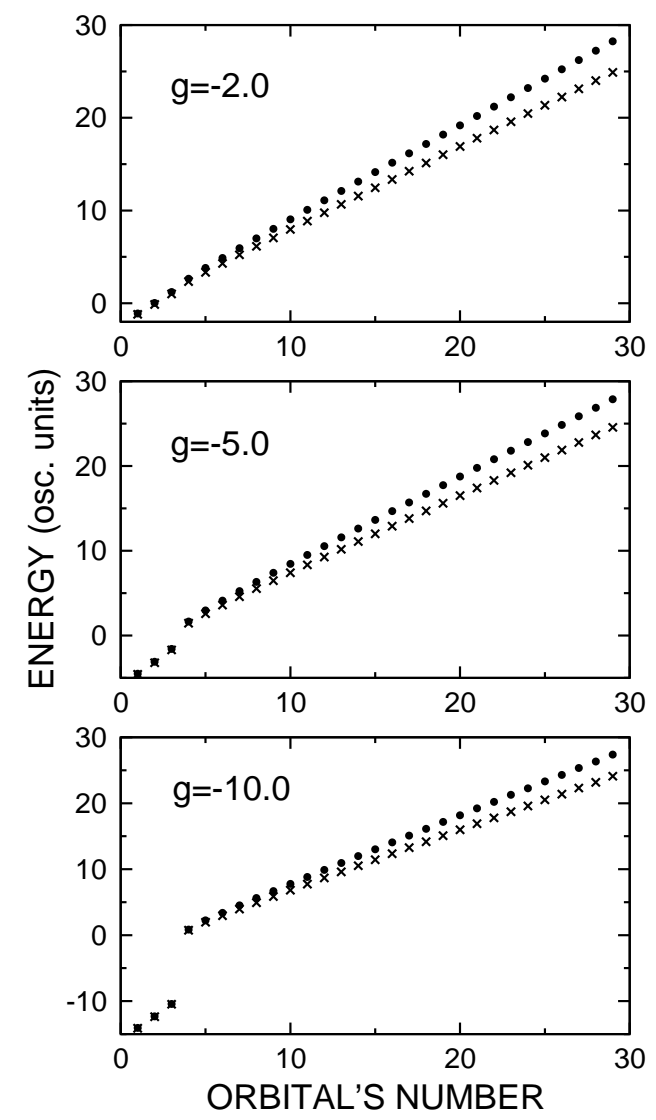

FIG. 7: Single-particle Hartree-Fock energies for various strength of attraction between atoms of different components. For strong enough attraction the energy gap is developed which separates off the populated levels. Dots and crosses correspond, respectively, to the first and second component.

in the lower line sum) ones represent the interaction energy between atoms within pairs whereas the last term describes the pairs interaction (i.e., the interaction between distinguishable atoms from different pairs).

Next, we minimize the expression (15) with respect to the real amplitudes $u_{n}, v_{n}$ under extra condition that the total number of pairs equals the number of atoms in each component $\sum_{n} v_{n}^{2}=N$. To this end, it is convenient to introduce new variables $\Theta_{n}$ in the following way: $u_{n}=$ $\sin \Theta_{n}$ and $v_{n}=\cos \Theta_{n}$. Now, the energy of the ground state $\mathrm{E}_{\mathrm{BCS}}$ is a function of angles $\Theta_{n}$ and its conditional minimum can be found by using the Lagrange multiplier technique. One introduces the many-variable function $\mathrm{F}_{\mathrm{BCS}}=\mathrm{E}_{\mathrm{BCS}}-\lambda G$ where $G=\sum_{n} \cos ^{2} \Theta_{n}-N$ and looks for the unconditional minimum of function $\mathrm{F}_{\mathrm{BCS}}$. The necessary condition for that turns, together with the normalization requirement for the total number of pairs, to the following set of equations

$$
\begin{aligned}
& \sum_{m \neq n} V_{n n, m m} \sin 2 \Theta_{m}-2 \tan 2 \Theta_{n} \sum_{m \neq n} V_{n m, n m} \cos ^{2} \Theta_{m} \\
& =\left(e_{n}^{(1)}+e_{n}^{(2)}+V_{n n, n n}-\lambda\right) \tan 2 \Theta_{n} \\
& n=0,1,2, \ldots
\end{aligned}
$$



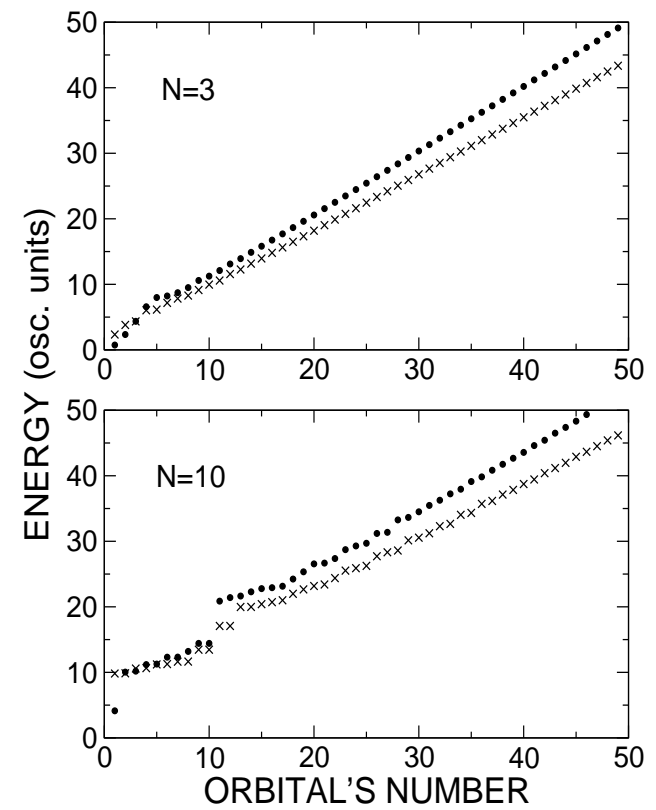

FIG. 8: Single-particle Hartree-Fock energies for $g=+10.0$ and $3+3$ atoms system (the upper frame). No energy gap is developed. The lower frame shows the same spectrum but in the case of $10+10$ atoms system in the excited state with antiferromagnetic phase (as in the lowest part in Fig. 4). Surprisingly, the energy gap is visible again although there are some states within it.

$$
\sum_{m} \cos ^{2} \Theta_{m}=N
$$

for variables $\Theta_{n}$ and the Lagrange multiplier $\lambda$. We solve numerically this set of equations and then check whether what we found is a minimum or not. We just randomly disturb obtained amplitudes $u_{n}, v_{n}$ and verify that the energy of the system according to expression (15) always gets higher.

There is an important issue of choosing the appropriate two-particle basis functions for pair states. It turns out that it is good to build the pair wave functions using single-particle Hartree-Fock orbitals. In such a case the ground state of the system involves relatively small number of pair states. In Fig. 6] we plot the energy of the system of $3+3$ atoms as a function of the coupling constant $g$, calculated within both the Hartree-Fock and BCS approaches. It is seen that for strong enough attraction $(g<-3)$ the ground state of the system becomes of BCS type. Simultaneously, the energy gap in a single-particle Hartree-Fock spectrum is being developed (see Fig. 2). It is favorable for the system to lower its energy by forming pairs of atoms of different components. Fig. 9] shows that, in fact, 50 states basis is already good enough. The densities for both fractions are plotted in the inset in Fig. [6 for $g=-10$. In BCS case the broad background is developed, not present for the Hartree-Fock densities. In both cases the fragmenta- tion pattern is visible which means that one component serves the other as a periodic potential. This resembles somewhat the situation when the atoms are confined in the optical lattice. For the interaction strength $g>-3$ one has $v_{n} \approx 1, u_{n} \approx 0$ for $n<3$ (and simultaneously $v_{n} \approx 0, u_{n} \approx 1$ for $n>3$ ) and there is no difference between the Hartree-Fock and BCS densities.

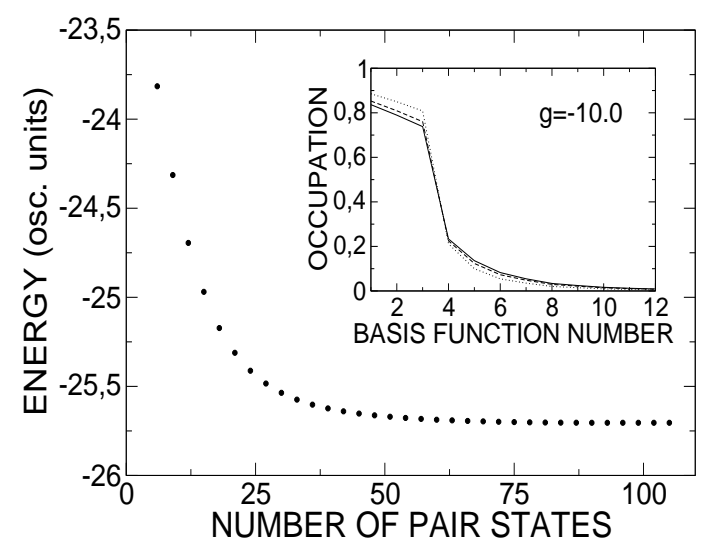

FIG. 9: BCS energy of $3+3$ atoms system as a function of number of states in the basis of pair states for $g=-10$. In this case $\mathrm{E}_{\mathrm{HF}}=-23.4$ whereas $\mathrm{E}_{\mathrm{BCS}}=-25.7$ osc. units. The inset shows corresponding occupations in cases when the number of basis states equals to 12 (dotted line), 21 (dashed line), and 105 (solid line).

According to definition (8) one can calculate the oneparticle density matrices for the BCS ground state given by (13)

$$
\begin{aligned}
& \rho_{1}^{(1)}\left(\mathbf{x}_{1} ; \mathbf{x}_{1}^{\prime}\right)=\sum_{n} v_{n}^{2} \varphi_{n}^{(1) *}\left(\mathbf{x}_{1}\right) \varphi_{n}^{(1)}\left(\mathbf{x}_{1}^{\prime}\right) \\
& \rho_{1}^{(2)}\left(\mathbf{y}_{1} ; \mathbf{y}_{1}^{\prime}\right)=\sum_{n} v_{n}^{2} \varphi_{n}^{(2) *}\left(\mathbf{y}_{1}\right) \varphi_{n}^{(2)}\left(\mathbf{y}_{1}^{\prime}\right)
\end{aligned}
$$

Assuming the energetically lowest $N$ pair states are fully occupied, i.e. $v_{n<N}=1, u_{n<N}=0$ and $v_{n \geq N}=$ $0, u_{n \geq N}=1$, the above formulas match the ones derived within the Hartree-Fock approach. The same is true also for the two-particle density matrix which is given by

$$
\begin{aligned}
& \rho_{2}^{(1)(2)}\left(\mathbf{x}_{1}, \mathbf{y}_{1} ; \mathbf{x}_{1}^{\prime}, \mathbf{y}_{1}^{\prime}\right)=\sum_{n, m}\left(u_{n} u_{m} v_{n} v_{m}+v_{n}^{2} v_{m}^{2}\right) \times \\
& \varphi_{n}^{(1) *}\left(\mathbf{x}_{1}\right) \varphi_{m}^{(2) *}\left(\mathbf{y}_{1}\right) \varphi_{n}^{(1)}\left(\mathbf{x}_{1}^{\prime}\right) \varphi_{m}^{(2)}\left(\mathbf{y}_{1}^{\prime}\right)
\end{aligned}
$$

In Fig. 10 we have shown the diagonal part of twoparticle density matrix for the BCS ground state of $3+3$ atoms system for the interaction strength $g=-10$. For this value of $g$ the pairing phenomenon is already well established and leads to $10 \%$ decrease in energy in comparison with the Hartree-Fock case. The characteristic crests along ' $x$ ' and 'y' axis are developed as a result of presence of higher $n$ (bigger size understood as the mean value of $|\mathbf{x}-\mathbf{y}|$ ) pair states in the BCS ground state (see also Fig. 6). No such crests are visible for the HartreeFock ,ground state". 


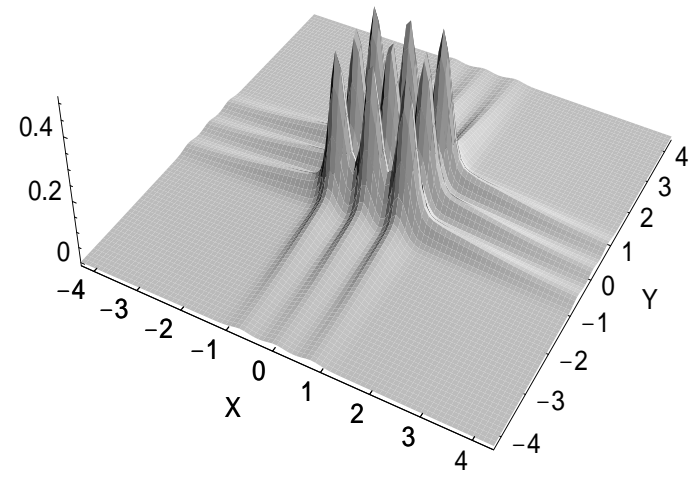

FIG. 10: Diagonal part of two-particle density matrix for the BCS ground state of $3+3$ atoms system, i.e., joined probability of detecting two distinguishable atoms. The interaction strength $g=-10$. All quantities are given in oscillatory units.

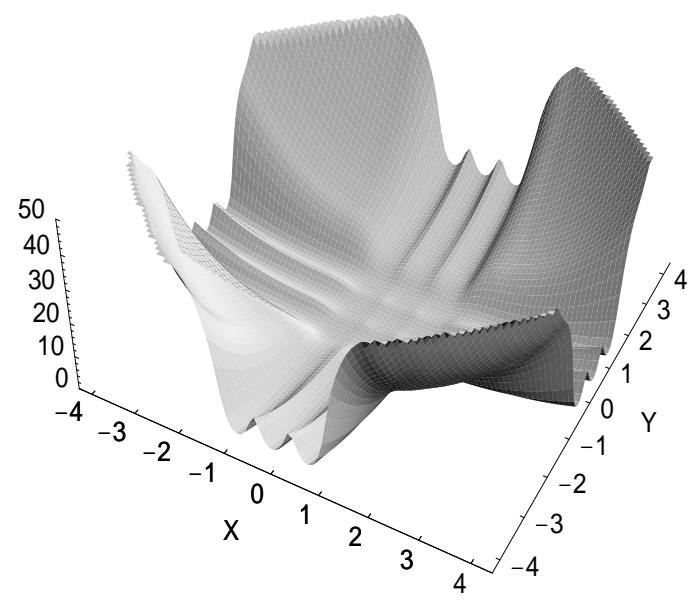

FIG. 11: Correlation function for the BCS ground state of $3+3$ atoms system. The interaction strength $g=-10$. Only this part of 'xy' plain is considered where the two-particle density matrix essentially differs from zero. All quantities are given in oscillatory units.

In Fig. 11] we plot the correlation function $g_{2}$, defined generally as

$$
g_{2}(\mathbf{x}, \mathbf{y})=\frac{\rho_{2}^{(1)(2)}(\mathbf{x}, \mathbf{y} ; \mathbf{x}, \mathbf{y})-\rho_{1}^{(1)}(\mathbf{x} ; \mathbf{x}) \rho_{1}^{(2)}(\mathbf{y} ; \mathbf{y})}{\rho_{1}^{(1)}(\mathbf{x} ; \mathbf{x}) \rho_{1}^{(2)}(\mathbf{y} ; \mathbf{y})}
$$

in the case of one-dimensional $3+3$ atoms system under the same parameters as in the case of Fig. 10 It is true that the correlation function differs from zero mainly along the directions $y=x$ and $y=-x$. Whereas the former is not surprising because densities of both fractions look the same (see inset in Fig. 6), the latter means that

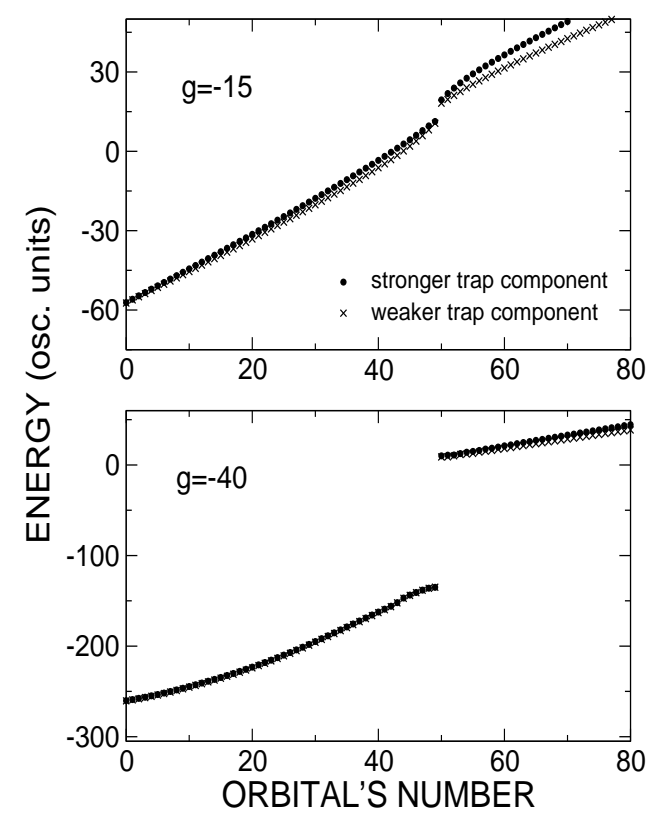

FIG. 12: Single-particle Hartree-Fock energies for various strengths of attraction between atoms of different components for a system of $50+50$ atoms. For strong enough attraction the energy gap is developed (upper frame) and the BCS ground state turns out to be reachable. When the interaction strength is increasing further the energy gap is getting bigger (lower frame).

the strong correlations are developed for distinguishable atoms being at positions $(2,4)$ and $(-4,-2)$ in oscillatory units. Since it corresponds to the size of pair states contributing essentially to the ground state of $3+3$ atoms system (approximately first 50 pair states - see Fig. 9) it means that the presence of $y=-x$ correlations supports the explanation of properties of the ground state with the help of pairing phenomenon.

Increasing the number of atoms in both fractions we find further qualitative changes in the properties of the ground state in comparison with $3+3$ case. When the coupling constant $g$ becomes smaller (i.e., for stronger attraction) the energy gap in the Hartree-Fock spectrum gets bigger (Fig. 12) just like in the case of $3+3$ system. However, some new features appear when one looks at the size of the pairs (Fig. 13) as well as at their energies (Fig. 14) for various values of the coupling constant $g$. For strong enough attraction the size of the pairs is getting frozen revealing plateau and the gap is developed in the energy spectrum. The energy gap in the pairs spectrum is perhaps the signature that the system enters a new phase characterized by the appearance of clusters of particles in its ground state, each built of four atoms.

It remains to be checked what is the range of validity of parameters used in our calculations. To this end, we show the following simple estimation. Binary collisions dominate provided the mean interparticle separation is much bigger than the s-wave scattering length, i.e., $n_{3 D}\left|a_{3 D}\right|^{3} \ll 1$. According to Olshanii's result [20], 


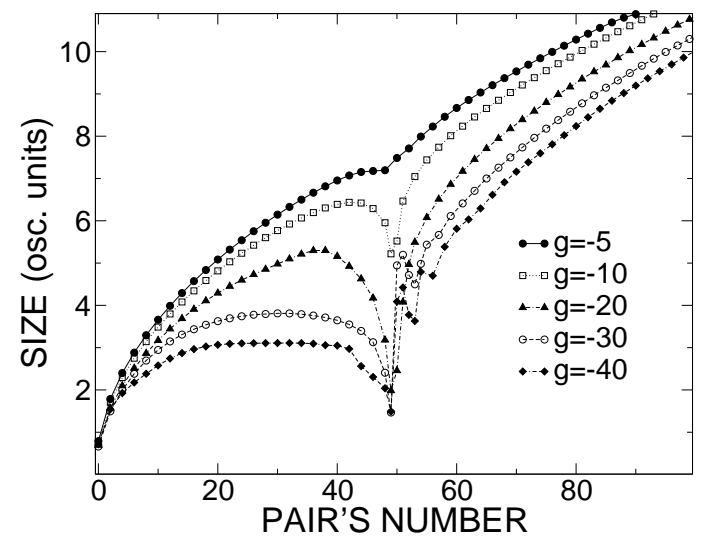

FIG. 13: Size of the pair states calculated as the mean value of $|\mathbf{x}-\mathbf{y}|$ for the $50+50$ atoms system. Note that for strong enough attraction the size is getting frozen and characteristic plateau is developed.

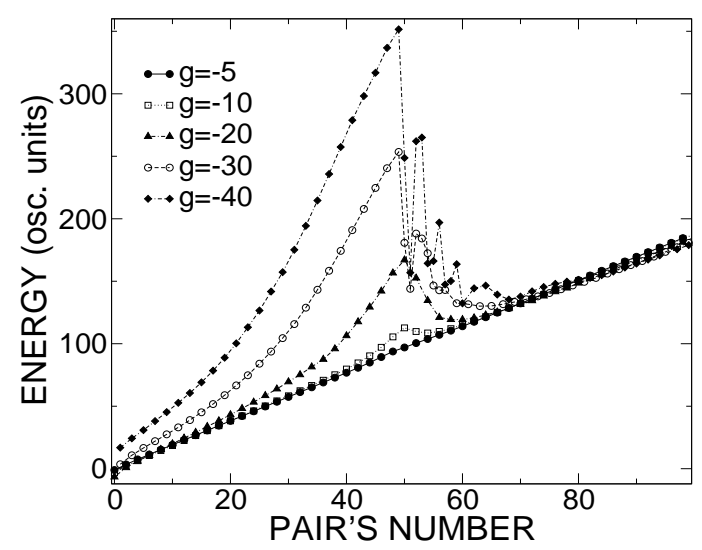

FIG. 14: The energy of the pair states for the system of $50+50$ atoms. For strong enough attraction the gap in the pairs spectrum is developed.

for elongated systems the effective one-dimensional interaction strength can be introduced via the relation

$$
g_{1 D}=\frac{g_{3 D}}{\pi a_{\perp}^{2}}\left(1-C \frac{a_{3 D}}{a_{\perp}}\right)^{-1}
$$

where $g_{3 D}=4 \pi \hbar^{2} a_{3 D} / m, C=1.4603$, and $a_{\perp}=$ $\left(\hbar / m \omega_{\perp}\right)^{1 / 2}$ determines the radial length scale. Assuming the tight confinement one can introduce onedimensional density in such a way that

$$
n_{3 D}=\frac{n_{1 D}}{\pi a_{\perp}^{2}} .
$$

The range of validity of our model can be then expressed as

$$
\frac{n_{1 D}\left|g_{1 D}\right|^{3} \alpha^{2}}{\pi\left|g_{1 D} C \alpha^{1 / 2}+4\right|^{3}} \ll 1
$$

where $\alpha=\omega_{z} / \omega_{\perp}$ and quantities $n_{1 D}$ and $g_{1 D}$ are already taken in units related to one-dimensional system. For example, for $\left|g_{1 D}\right|=10$ the condition (23) leads to the relation $\alpha \ll 0.075$ (or $\omega_{\perp} / \omega_{z} \gg 13$ ) which, first, acts in the same direction as the assumptions leading to the formula (21) and, second, can be fulfilled experimentally although for larger $\left|g_{1 D}\right|$ the radial squeezing is getting stronger and stronger.

\section{CONCLUSIONS}

We have analyzed the system of two-component Fermi gas confined in a magnetic trap at zero temperature under assumption that the only interparticle interaction left is the repulsion or attraction between atoms of different components. We employ the microscopic description of the system by using the explicit atomic wave functions. The structure of the ground state strongly depends on the sign of the coupling constant. When distinguishable atoms repel each other strongly enough both fractions separate with that which is confined stronger filling the center of the trap. In the opposite case, i.e., when the atoms strongly attract each other, the ground state dramatically changes its character. The BCS pairs appear which is proved by showing that the energy of the system of pairs is lower than the corresponding HartreeFock energy and by analyzing the two-particle correlation function. For even stronger attraction the system enters a new phase where presumably clusters of particles are formed. We are working currently on extension of these calculations to the three-dimensional case as well as on inclusion of the optical lattice and finite temperatures.

\section{Acknowledgments}

M.B. acknowledges support by the Polish KBN Grant No. 2 P03B 052 24. T.K. and K.R. were supported by the Polish Ministry of Scientific Research Grant Quantum Information and Quantum Engineering No. PBZ-MIN008/P03/2003.
[1] B. DeMarco and D.S. Jin, Science 285, 1703 (1999).

[2] A.G. Truscott, K.E. Strecker, W.I. McAlexander, G.B. Partridge, and R.G. Hulet, Science 291, 2570 (2001); F. Schreck, L. Khaykovich, K.L. Corwin, G. Ferrari, T. Bourdel, J. Cubizolles, and C. Salomon, Phys. Rev. Lett.
87, 080403 (2001); S.R. Granade, M.E. Gehm, K.M. O'Hara, and J.E. Thomas, Phys. Rev. Lett. 88, 120405 (2002).

[3] Z. Hadzibabic, C.A. Stan, K. Dieckmann, S. Gupta, M.W. Zwierlein, A. Görlitz, and W. Ketterle, Phys. Rev. 
Lett. 88, 160401 (2002).

[4] G. Roati, F. Riboli, G. Modugno, and M. Inguscio, Phys. Rev. Lett. 89, 150403 (2002).

[5] D.A. Butts and D.S. Rokhsar, Phys. Rev. A 55, 4346 (1997).

[6] K. Góral, B.-G. Englert, and K. Rza̧żewski, Phys. Rev. A 63, 033606 (2001).

[7] G.M. Bruun and K. Burnett, Phys. Rev. A 58, 2427 (1998).

[8] G.M. Bruun and C.W. Clark, , Phys. Rev. Lett. 83, 5415 (1999).

[9] L. Vichi and S. Stringari, Phys. Rev. A 60, 4734 (1999).

[10] T. Karpiuk, M. Brewczyk, and K. Rzążewski, J. Phys. B 35, L315 (2002); T. Karpiuk, M. Brewczyk, Ł. Dobrek, M.A. Baranov, M. Lewenstein, and K. Rzążewski, Phys. Rev. A 66, 023612 (2002); T. Karpiuk, M. Brewczyk, and K. Rzążewski, J. Phys. B 36, L69 (2003).

[11] M. Cozzini and S. Stringari, cond-mat/0305268

[12] M. Holland, S.J.J.M.F. Kokkelmans, M.L. Chiofalo, and
R. Walser, Phys. Rev. Lett. 87, 120406 (2001); M.L. Chiofalo, S.J.J.M.F. Kokkelmans, J.N. Milstein, M.J. Holland, Phys. Rev. Lett. 88, 090402 (2002).

[13] W. Hofstetter, J.I. Cirac, P. Zoller, E. Demler, and M.D. Lukin, Phys. Rev. Lett. 89, 220407 (2002).

[14] J.L. Bohn, Phys. Rev. A 61, 053409 (2000).

[15] M.A. Baranov, M.S. Mar'enko, Val.S. Rychkov, and G.V. Shlyapnikov, Phys. Rev. A 66, 013606 (2002).

[16] T.L. Ho and V.B. Shenoy, Phys. Rev. Lett. 77, 3276 (1996); H. Pu and N.P. Bigelow, Phys. Rev. Lett. 80, 1130 (1998).

[17] M. Trippenbach, K. Góral, K. Rzążewski, B. Malomed, and Y.B. Band, J. Phys. B 33, 4017 (2000).

[18] R. Roth and H. Feldmeier, J. Phys. B 34, 4629 (2001).

[19] J.M. Eisenberg and W. Greiner, Microscopic Theory of the Nucleus (North-Holland, Amsterdam, 1972).

[20] M. Olshanii, Phys. Rev. Lett. 81, 938 (1998). 